\title{
Salbe erhält Status als Medizinprodukt
}

Seit nunmehr 10 Jahren erfreuen sich die Deumavan ${ }^{\circledast}$ Intimpflegeprodukte einer großen Nachfrage. Diese Salben zur kosmetischen Pflege wurden über die Jahre hinsichtlich zahlreicher medizinischer Fragestellungen klinisch getestet. Die Ergebnisse führten Mitte 2017 dazu, dass die Intimpflegesalben den Status als Medizinprodukte erhielten und nun als Deumavan ${ }^{\circledast}$ Schutzsalbe (Neutral und Lavendel) erhältlich sind.
Die umfangreichen klinischen Patientenergebnisse verschiedener Erkrankungen der Frau und des Mannes zeigen, dass die Intimpflegesalbe zur Pflege des äußeren Intimbereiches und zur Schmerzund Juckreizlinderung eingesetzt werden kann. In Zusammensetzung unterscheiden sich die früheren Kosmetikprodukte Deumavan $^{\otimes}$ Intimpflegesalbe und die neuen Medizinprodukte Deumavan ${ }^{\infty}$ Schutzsalbe nicht.
Beide Salben sind in verschiedenen Größen und Darreichungsformen (Tuben und Dosen) verfügbar. Die Schutzsalbe (Neutral und Lavendel) unterstützt die natürlichen Hautfunktionen des gesamten Intimbereichs. Die neutrale Schutzsalbe ist frei von Zusätzen wie Konservierungsstoffen, Emulgatoren, Duftstoffen und Hormonen. Zur Reinigung, speziell des Intimbereichs, wird das Kosmetikum Deumavan $^{\circledast}$ Waschlotion sensitiv (Neutral bzw. Lavendel) angeboten. Weitere Informationen sind über die Webseite www.deumavan.com abrufbar.

Nach Informationen von Kaymogyn

Hyaluronfiller

\section{Anatomiekenntnisse ermöglichen individuelle Behandlungen}

Die minimalinvasive ästhetische Medizin unterliegt einem ständigen Wandel. Patienten, die gesunde Haut und eine natürliche Ausstrahlung anstreben, ohne dabei ihre eigene Persönlichkeit zu verändern, beanspruchen zunehmend individuelle Behandlungsprogramme. Fundierte Anatomiekenntnisse unterstützen den Behandler dabei, Behandlungen an Patientenwünsche anzupassen.

Während des diesjährigen Ästhetiktages in Düsseldorf stand die Behandlung mit Hyaluronfillern im Vorder- grund. Die Teilnehmer erhielten tiefe Einblicke in die Gesichtsanatomie anhand von Live-Demonstrationen am Patienten. Experten gaben während der Injektionen Tipps und praktische Tricks für eine präventive und kurative Behandlung.

Dr. Sopena, Facharzt für Dermatologie aus Spanien, demonstrierte beispielsweise an einem männlichen Patienten die Besonderheiten bei der Injektion von Hyaluronfillern in die Stirn. Dabei wurde besonders auf ver- schiedene Zugangsmöglichkeiten für die Injektion eingegangen und wie der individuelle Gefäßverlauf des Patienten diese beeinflusst.

Für Ärzte, die nicht vor Ort waren, werden alle sieben Live-Behandlungen mit anatomischen Hintergrundinformationen in Form von Kasuistiken veröffentlicht. Mit Gegenüberstellungen von Anatomieabbildungen und Fotos der Patienten sowie detaillierten Hintergrundinformationen werden die Live-Behandlungen nachvollziehbar gemacht. Diese werden außerdem auf dem Galderma Behandlerportal exklusiv zur Verfügung gestellt.

Weitere Informationen finden Sie unter https://www.galderma-aesthetik.de.

Nach Informationen von Galderma

\section{Neue Generation Urea}

\section{Pflege für trockene Füße}

Ein feuchtigkeitsspendendes Gel-Oil mit $10 \%$ Urea ISDIN $^{\circledR}$ ist Teil der von ISDIN geschaffenen „Neuen Generation Urea“. Diese zeichnet sich durch die Urea ISDIN ${ }^{\circledR}$ Formulierung aus: So wird die Barrierefunktion der Haut sowie auch das Immunsystem verbessert. Die Stimulation der Kollagensynthese verbessert zudem die mechanische Schutzfunktion, Konsistenz und Flexibilität der Haut.

Mit Ureadin ${ }^{\circledast}$ Podos werden spröde und raue Stellen sowie Hornhaut und
Schrunden dank der exfolierenden Wirkung von Milchsäure und Bacillus Ferment beseitigt. Die hautregenerierenden Inhaltsstoffe Allantoin und Panthenol sowie Sheabutter lindern Trockenheit und Risse.

Auch die Wirksamkeit ist klinisch belegt: So konnte bei $97 \%$ der Anwendungen nach 14 Tagen ein deutlich besserer Hautzustand an den Fersen festgestellt werden. Ausgezeichnet wird Ureadin ${ }^{\circledast}$ Podos zudem durch eine einzigartige Gel-Oil-Textur: Sie ist frisch und leicht, nicht fettend und zieht schnell ein - ideal für trockene Füße.

Ergänzt wird das Portfolio durch Ureadin ${ }^{\circledast}$ Podos Db, das speziell für empfindliche diabetische Füße entwickelt wurde. Die dort enthaltenen $5 \%$ Urea ISDIN ${ }^{\circledR}$ spenden Feuchtigkeit, Polidocanol lindert außerdem den Juckreiz. Ureadin ${ }^{\bowtie}$ Podos Db hilft das Risiko für Ulzerationen zu verringern, verbessert Mikrozirkulation sowie die Hautelastizität. Dank Hagebutte und BioEcolia $^{\circledR}$ hilft es außerdem bei der natürlichen Reparatur der Haut und dabei künftige Hautprobleme zu vermeiden.

Nach Informationen von ISDIN 